\title{
PACKING ENERGY AND IRON TO SERVE THE MELTSHOP REQUIREMENTS*
}

\author{
Alessandro Martinis ${ }^{1}$ \\ Daniela Dalle Nogare ${ }^{1}$ \\ Annamaria Volpatti ${ }^{1}$ \\ Joel Morales ${ }^{2}$
}

\begin{abstract}
The performance requirements for modern steelmaking facilities are ever more stringent, as customers and markets are continuously pushing for technologies which allow having high quality steel produced in an economic and sustainable way. In the details, this paper has the aim to present the excellent results obtained by integrated steel plant where an EAF is fed with high quality hot ENERGIRON DRI. The recent technology developments, combined with the affirmed basic process design, contributed to further maximize the energy of the DRI, reaching the EAF at temperatures above $600^{\circ} \mathrm{C}$ with a carbon content up to $3.5 \%$, as confirmed by plant data. These improved input material characteristics resulted in reduced electrical energy consumption and increased liquid steel production.
\end{abstract}

Keywords: Direct reduction technology; High temperature DRI; High carbon DRI. 


\section{INTRODUCTION}

The performance requirements for modern steelmaking facilities are ever more stringent, as customers and markets are continuously pushing for technologies which allow having high quality steel produced in an economic and sustainable way.

The BF-BOF, using directly iron ore as raw material for steel production, is for sure a change for producing high grade steel; nevertheless, using coal as reducing agent, the significant amount of $\mathrm{CO}_{2}$ emitted in the atmosphere as byproduct of the reduction reactions, combined with the low energy efficiency of this process, contributes to make this route often incompatible with restrictions imposed by environmental regulations and less competitive.

On the contrary, when steel is produced via EAF charged with 100 percent scrap, the environmental parameters are more easily respected but, due to scrap availability and quality (market price fluctuations and several residual elements and nitrogen level), price and grade of the final steel are not competitive: for this reason the EAF operators intensified the search for alternative iron materials to be fed to the EAF. Using DRI or $\mathrm{HBI}$ as feed for the EAF, mills can produce the superior steel grades demanded by such users as automotive, special and tool steels that were originally only possible in integrated mills with BF-BOF. So in terms of quality, the DR-EAF route is certainly not limited to producing simply commercial steel grades. Add to this the ability to now produce DRI in capacities exceeding 2 million tons per year, and coupling this type of plant with a modern EAF meltshop, a new type of "integrated" mill for the near future is available: a high volume, integrated DR-EAF mill, capable of competing in both quality and cost.

This is possible only in integrated steel plant where an EAF is fed with high quality hot DRI. The recent Energiron technology developments, combined with the affirmed basic process design, contributed to further maximize the energy of the produced $\mathrm{DRI}$, reaching the EAF at temperatures above $600^{\circ} \mathrm{C}$ with carbon content up to $3.5 \%$, as confirmed by plant data. These improved input material characteristics resulted in reduced electrical energy consumption and increased liquid steel production.

\section{DISCUSSION}

\subsection{Energiron DRP Basic Process Scheme}

The chemical composition of the DRI (in particular degree of metallization and carbon content) combined with its temperature determines such important factors as yield, slag weight, energy consumption, carbon and raw material feeding rates and oxygen usage.

The goal of the Energiron technology, jointly developed by Tenova and Danieli, is to produce DRI with metallization above $94 \%$ (reaching also 96\%) at carbon content easily controlled in the range between 2 and $4 \%$, as required by the meltshop, simply acting on the process parameters. In addition, when material is hot discharged from the reactor, DRI can be fed at the EAF directly at high temperatures.

In the detail, the Energiron DRP converts iron ore into metallic iron by means of hot $\left(>1080^{\circ} \mathrm{C}\right)$ reducing gases that flow in the direction opposite the solid material inside the moving bed shaft furnace (reactor) operated at a pressure of approximately 6 barg on the top. In the innovative Zero Reformer configuration, the Natural Gas, used as a reducing agent make up, is directly fed inside the reactor where is converted into reformed gas simply by exploiting the catalytic power of the DRI. 
Since the reducing gases are generated in the reduction section, the overall energy efficiency of the direct reduction is optimized and most of the energy supplied to the process is taken by the product with minimum energy losses to the environment: as compared to other processes for which the overall efficiency is below $80 \%$, for this scheme the efficiency is around $86 \%$. This arrangement ultimately turns into very low natural gas consumption, even lower than $2.35 \mathrm{Gcal} / \mathrm{t}$.

Nevertheless this basic reduction scheme is unchanged also when an external reducing gas is available (Syngas from coal gasification or produced in an external reformer, COG, etc.): this is a key feature of the Energiron DRI technology.

A brief summary of the process configuration is as follows. Oxygen is therefore removed from the iron ore by chemical reactions based on hydrogen (H2) and carbon monoxide (CO) for the production of highly metallized DRI.

The exhaust reducing gas (top gas) leaves the reactor at about $400-450^{\circ} \mathrm{C}$ and it is subsequently treated in order to clean it and get rid of the oxidant elements $(\mathrm{H} 2 \mathrm{O}$ and CO2) generated by the reduction reactions. In particular the top gas passes through the top gas heat recuperator (heat exchanger), where its energy is recovered to produce steam, before passing through the quenching/scrubbing system. In these units, water is condensed and easily removed from the gas stream together with some dust carried by the gas. Subsequently the scrubbed gas, after being compressed, is treated in the $\mathrm{CO} 2$ removal unit where $\mathrm{CO} 2$, byproduct of reduction reactions, is selectively removed. The presence of such a $\mathrm{CO} 2$ removal system, intrinsically included in the basic reduction circuit, Energiron DRP is the lowest carbon footprint of any iron-making technology, with the additional advantage that selectively removed $\mathrm{CO} 2$ can be sold for subsequent further profits.

From the environmental impact point of view, the selective elimination of both byproducts generated by the reduction process, namely water $(\mathrm{H} 2 \mathrm{O})$ and carbon dioxide (CO2), which are eliminated through the top gas scrubbing and the $\mathrm{CO} 2$ removal system respectively, is one of the inherent and most important features of the Energiron process.

The upgraded gas is finally mixed with the natural gas make-up before passing first through an humidifier, where water content in the gas is regulated, and later through an heater for increasing temperature up to $950^{\circ} \mathrm{C}$, thus closing the reducing gas circuit (Figure 1). Direct internal combustion on the reducing gas by means of oxygen injection finally tunes the gas temperature to the desired value at Reactor inlet.

The continuous gravity flow of the material through the reduction furnace is regulated by a rotary valve located at the bottom of the vessel. Specially designed flow feeders ensure the uniform flow of solids within the shaft. DRI is finally discharged, hot or cold, by automated mechanisms, consisting of pressurized bins and special valves. 


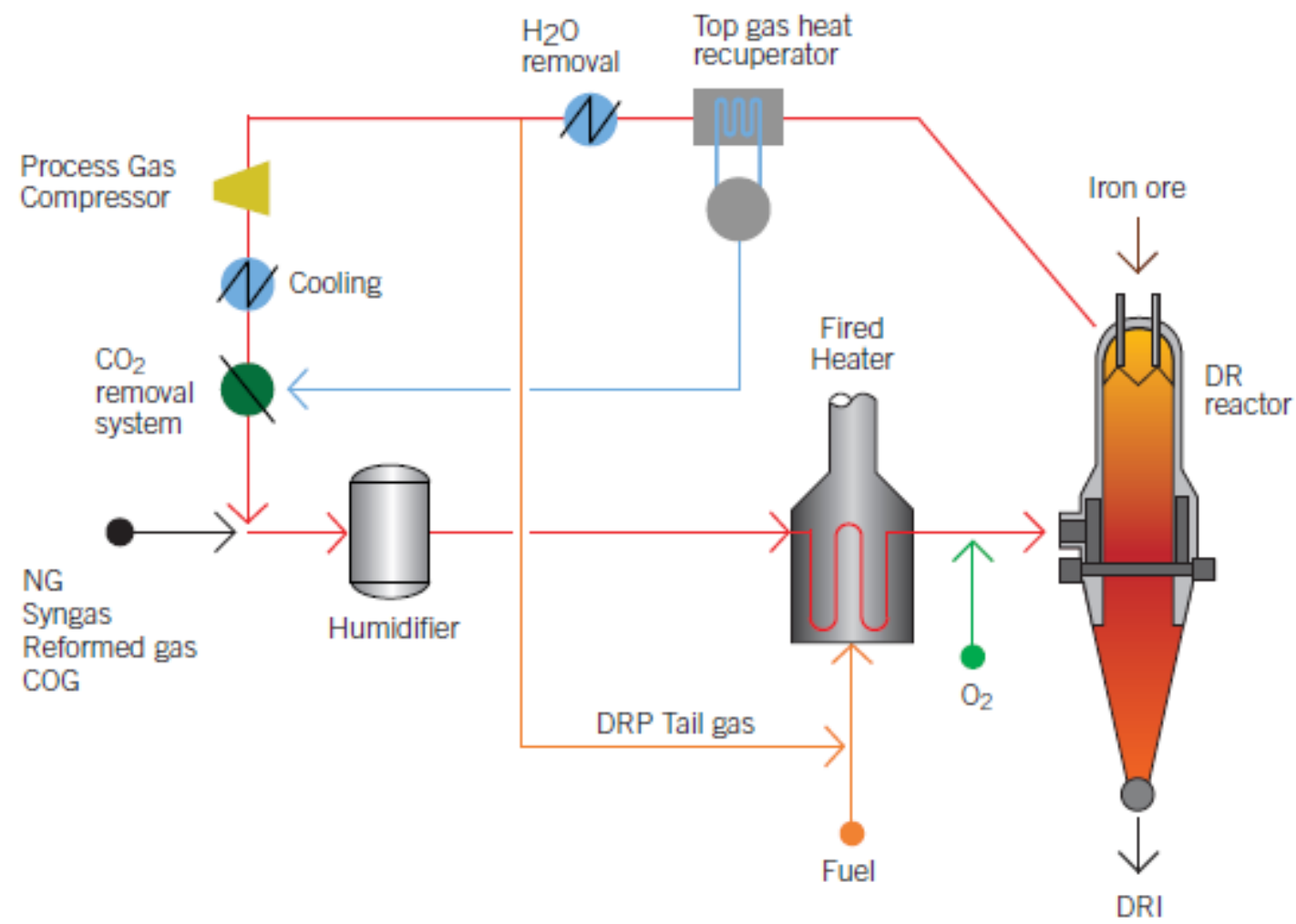

Figure 1. Energiron technology - reduction circuit scheme

Once cold DRI is selected as Reactor product, cooling gas is fed to the lower conical part of the furnace at about $40^{\circ} \mathrm{C}$, flowing upward countercurrent to the DRI moving bed, and is extracted at the top of the cone. Natural gas is injected as make-up to the cooling gas circuit for optimum efficiency and control of the cooling and carburization processes.

Once instead Hot DRI is discharged from the reactor, thanks to the HYTEMP pneumatic transport system, the hot discharged DRI reaches the EAF at temperatures above $600^{\circ} \mathrm{C}$. Alternatively, in case of a stop of the EAF, the hot DRI can be cooled in an external cooling vessel or, for plants equipped with dual reactor (as Suez), directly inside the reactor cone by passing a cooling gas flow.

The system operates by using a transport gas (either inert gas or the process gas itself) to carry the hot DRI through a pneumatic pipe to a holding bin above the electric furnace. The transport gas is removed from the circuit and recycled back to the DR plant and the hot DRI is charged to the holding bin for continuous feeding to the EAF. 

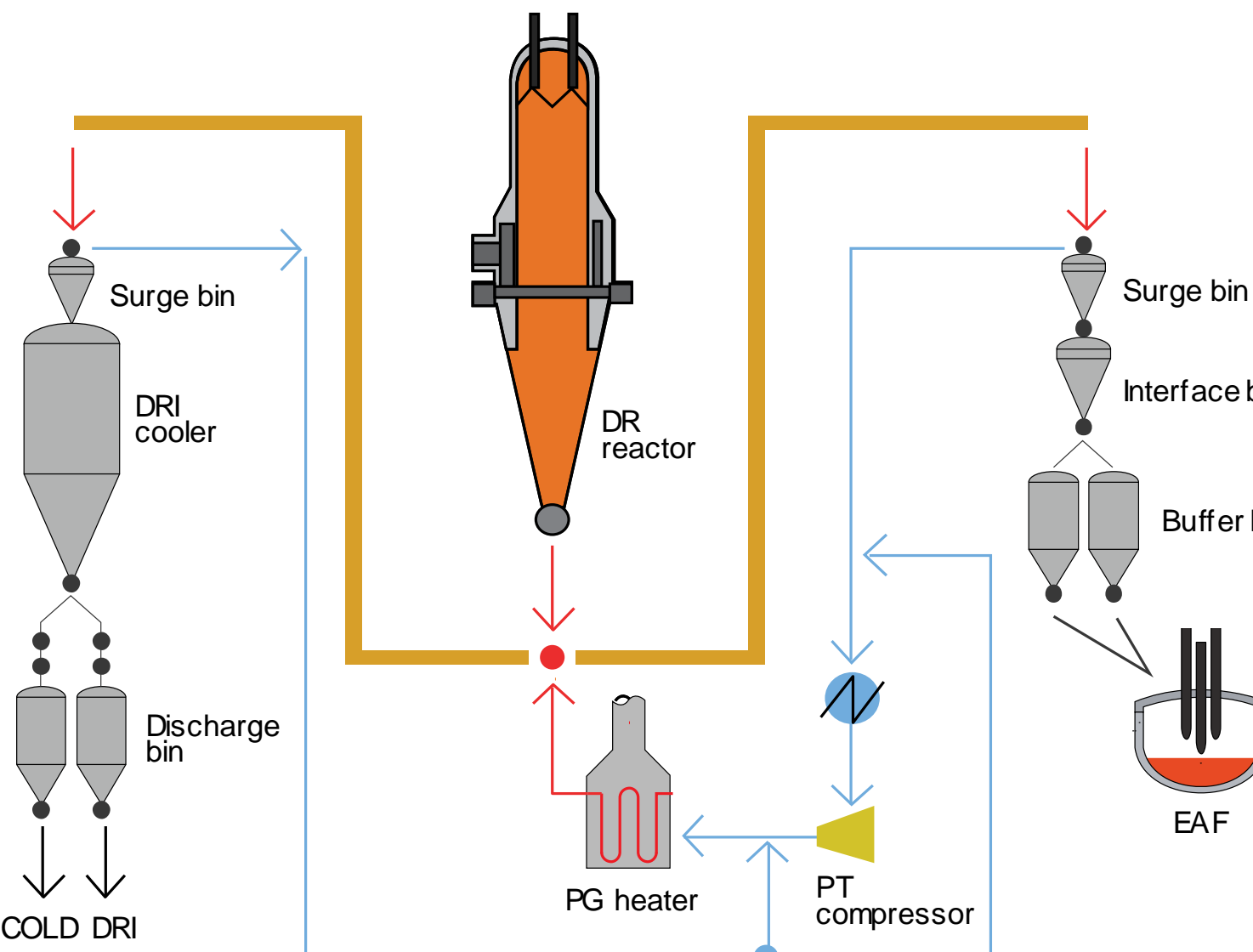

Carrier gas make up

Figure 2. HYTEMP system scheme offers the most adequate arrangement for integrated steelmaking facilities

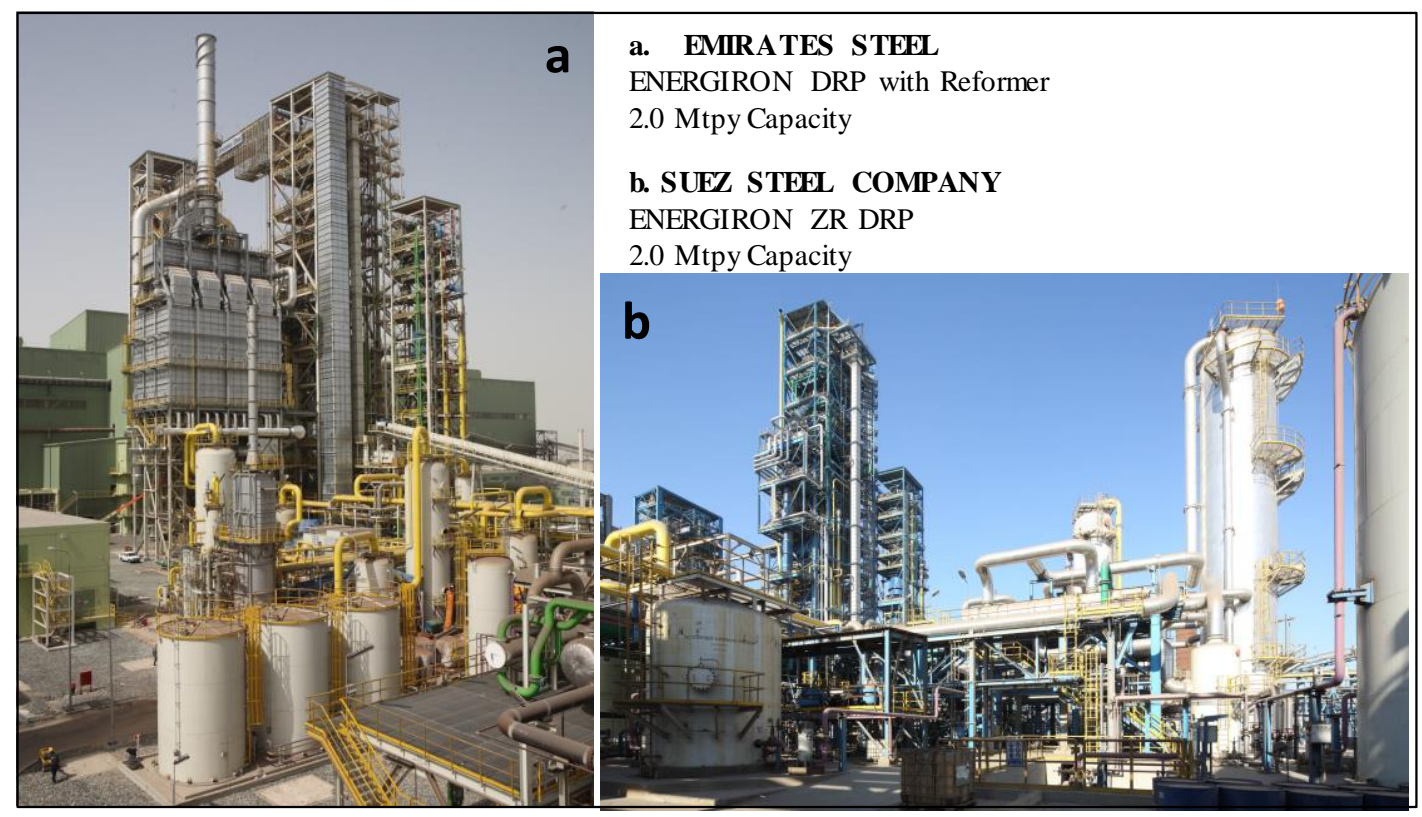

Figure 3. Energiron DRP - ES and SSC 


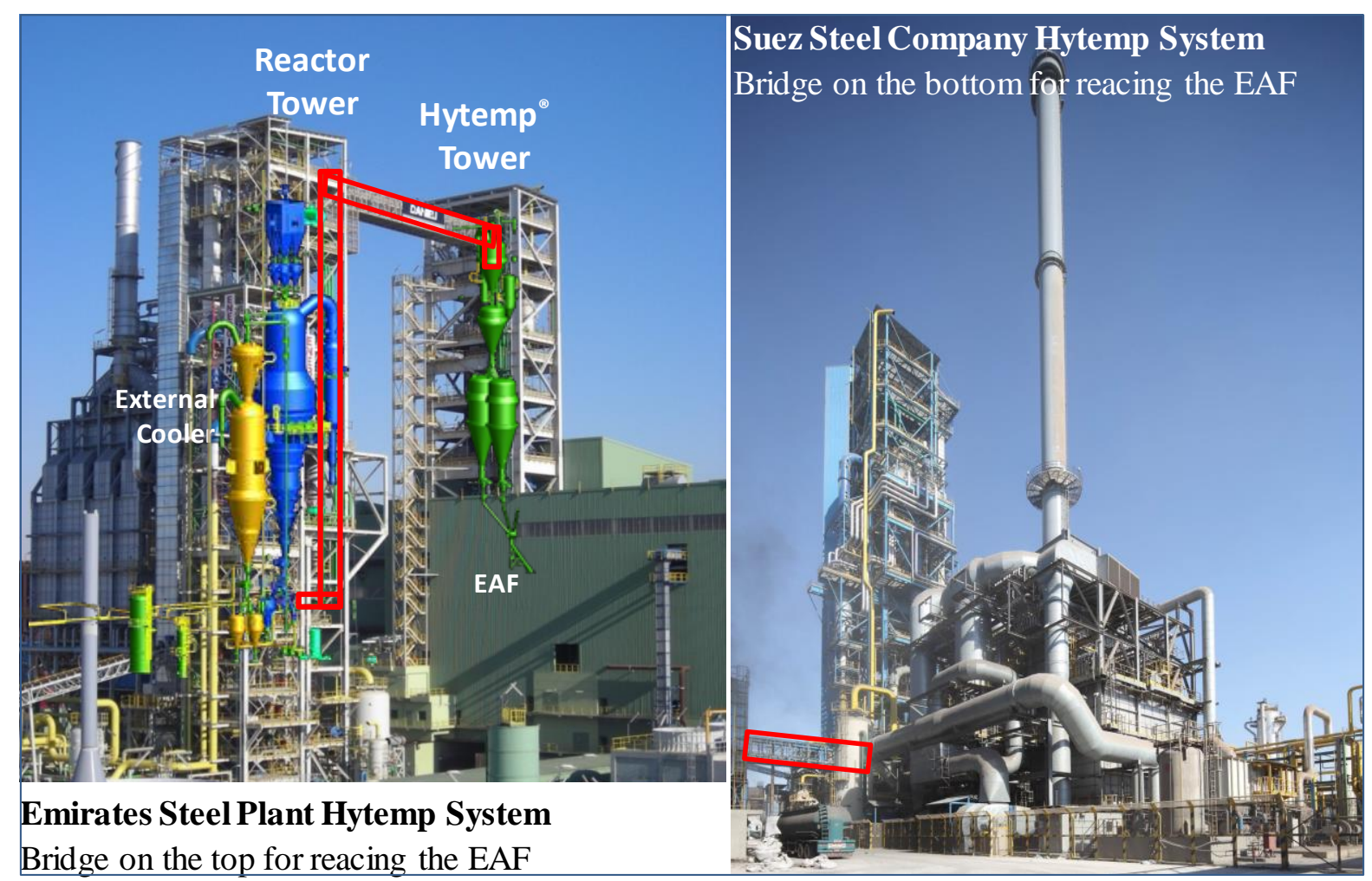

Figure 4. HYTEMP SYSTEM- ES and SSC

\subsection{Carbon Content in the DRI}

As previously mentioned, carbon in the EAF is required to reduce the remaining $\mathrm{FeO}$ in the DRI. For this reason, when using high percentages of DRI in the metallic charge, the carbon content in the bath has to be set according to the meltshop needs to maximise meltshop productivity. At this regard, the best solution for reaching required carbon content in the EAF is feeding the DRI with desired amount of carbon in the form of combined carbon, thus minimizing external carbon (graphite) additions. This combined carbon, in the form of cementite $\mathrm{Fe} 3 \mathrm{C}$, is more efficiently and completely used in the EAF: as a matter of fact, while coal and graphite additions to the EAF give yields of $40 \%$ or less, due to particle blow-off and the content of ash and other materials in coal, the combined carbon in DRI has a yield of $100 \%$. Furthermore, the conversion of $\mathrm{Fe} 3 \mathrm{C}$ into iron and carbon is an exothermic reaction which further improves the thermal efficiency in the EAF thus decreasing electric power requirements and promoting easy foamy slag generation; in other words it is like adding chemical energy to the EAF.

Traditionally DRI products have carbon levels around $1.3 \%$ or less; on the contrary Energiron DRI can be produced with a wide range of carbon content simply acting on few process parameters: Energiron DRI carbon levels can be selected in the range of $1.5-4.0 \%$ (as an example with the ZR Energiron configuration, optimum results are reached in the range of $3.0-3.5 \%$ and metallization of $>94 \%$ ).

In addition the high iron carbide content, being concentrated in the outer part of the reduced pellets, contributes to form a kind of "shell" which helps prevent the reoxidation providing a unique stability characteristic to this product. Energiron $\mathrm{DRI}$ is safe to transport and store under normal established procedures, eliminating the need for expensive briquetting operations. 
Basically the DRI carburization is the result of the following reactions:

1) $\mathrm{CO}+\mathrm{H}_{2} \Leftrightarrow \mathrm{C}+\mathrm{H}_{2} \mathrm{O}$

2) $2 \mathrm{CO} \Leftrightarrow \mathrm{C}+\mathrm{CO}_{2}$

3) $\mathrm{CH}_{4} \Leftrightarrow \mathrm{C}+2 \mathrm{H}_{2}$

1) $\Delta H_{25^{\circ} \mathrm{C}}=-131.84 \mathrm{~kJ} / \mathrm{kmol}$

2) $\Delta H_{25^{\circ} \mathrm{C}}=-173.67 \mathrm{~kJ} / \mathrm{kmol}$

3) $\Delta H_{25^{\circ} \mathrm{C}}=74.52 \mathrm{~kJ} / \mathrm{kmol}$

1) Beggs Reaction

2) Boudouard Reaction

3) Methane Cracking

Carburization by $\mathrm{CO}$ is exothermic and occurring only in case of high $\mathrm{CO}$ concentration. Its equilibrium is favored by low temperatures, but below $500^{\circ} \mathrm{C}$ its kinetic is very low. Carburization by cracking is instead endothermic and favored by high concentration of $\mathrm{CH} 4$ in the gas. Generally speaking, deposition of carbon by cracking is preferable because it releases hydrogen as product, while deposition by $\mathrm{CO}$ produces oxidants like $\mathrm{H} 2 \mathrm{O}$ and $\mathrm{CO} 2$.

In the ENERGIRON DRP most part of the carbon comes from cracking which, as already mentioned, is also beneficial for reduction because it releases hydrogen as product. Normally, carbon deposition occurs in the upper part of the Reactor cone or in the whole cone in case of hot discharge reactors; in both cases, the high methane (and heavier hydrocarbons) concentration together with high temperature promote the development of cracking. Indeed, metallic iron acts as a catalyst of the hydrocarbons cracking reaction, thus as soon as the hot and highly metallized sponge iron comes in contact with methane or higher hydrocarbons, the kinetics are quite fast. However, carbon deposition cannot occur indefinitely, because carbon hinders the cracking kinetics, as it supposedly forms a diffusion barrier that could prevent the access of the reducing gas (only hydrogen can partly penetrate it) and of hydrocarbons themselves [1]. This is generally not an issue for the reduction process because in the reactor the carburization zone is located below the metallization zone and the DRI metallization is completed at the time the pellet undergoes carburization. Moreover, since the carburization stops at certain extent, defined by the carburization zone conditions, the DRI transits through the cone of the reactor without major modifications from the reducing zone to the discharge section, nor in composition neither in temperature. This is a fortunate characteristic because the reactor can be seen as three reactors in series, a cylindrical zone for the reduction reactions, an isobaric zone for the carburization reaction, a cone for cooling or discharging. In these three reactor sections local conditions determine each particular aspect of the product almost independently (metallization, carburization, discharge temperature etc). In particular, the carburization occurring at high temperature leads to a high selectivity to cementite, thermodynamically favored by temperature, unlike that occurring at low temperature, were graphite is preferred. There might be a concern for the meta-stability of cementite, which naturally decomposes to graphite. However, the presence of sulfur in traces, both in the bustle gas and in the cone gas, stabilizes the cementite. Sulfur is adsorbed on iron very strongly, according to: 
$\mathrm{H} 2 \mathrm{~S} \Leftrightarrow \mathrm{H} 2+\mathrm{S}(\mathrm{ad}) \quad \Delta \mathrm{H} 25^{\circ} \mathrm{C}=-200 \mathrm{~kJ} / \mathrm{kmol}$

Already at relatively low sulfur activities aS $=\mathrm{pH} 2 \mathrm{~S} / \mathrm{pH} 2$ the coverage with $\mathrm{S}(\mathrm{ad})$ approaches a monolayer, e.g. at $850{ }^{\circ} \mathrm{C}$ at pH2S/pH2 $\approx 10-6[2,3]$. To nucleate graphite, an ensemble of free sites is necessary but with a high surface coverage of sulfur cementite decomposition cannot start.

\section{$\mathrm{Fe} 3 \mathrm{C} \Leftrightarrow 3 \mathrm{Fe}+\mathrm{C} \quad \Delta \mathrm{H} 25^{\circ} \mathrm{C}=-25.10 \mathrm{~kJ} / \mathrm{kmol}$}

If carburization mainly occurs in the zone wetted by the gas with a higher carburizing potential (natural gas/cone gas), a lower contribution also comes from the bustle gas, that can have some carburizing potential as well. With an external reformer, methane content in bustle gas is lower than $10 \%$ (vol), and carburization in the bustle zone gives a minor contribution to the total carbon deposition. On the other hand, in ZR plants, a quantitative carburization occurs also in the bustle area, due to the high concentration of methane, which is intrinsic to this process. The simulation of the DRP reactor was performed, for the Emirates Steel (Abu Dhabi) and Suez Steel Company (Suez) [5] DRP shaft reactors, by means of a CFD model developed by Danieli Research Center in conjunction with the University of Padova [4]. In Figure the maps of methane (panel A) and carbon (panel B) are shown for the two different reactors, simulated in hot discharge. ES DRP includes an external reformer while SSC is a ZR-process. Although the SSC reactor can operate in both configurations, i.e. hot and cold discharge, the hot discharge was chosen to allow a closer comparison with the ES hot discharge reactor. In panel A) the inlet streams are highlighted by the red arrow (reducing bustle gas) and blue arrow (natural gas). In the reactor cone methane composition is over $90 \%$ in both reactors. Near the entrance of the bustle gas methane composition is around $8 \%$ and $20 \%$ respectively for ES and SSC, and in the cylindrical zone the composition decreases to ca. $5 \%$ and $10 \%$ respectively, due to the auto-reforming and cracking reactions. In panel B) the map of carbon is shown for both reactors, together with a contour map representing the relative intensity of the methane cracking reaction. The red contour indicates the location of the strongest activity of methane cracking, that in ES is only at the interface between the bustle zone and the natural gas zone, where the hot metallized DRI comes in touch with natural gas, but in SSC is also in the zone near the entrance of the bustle gas, at high methane concentration. The mean carbon content of DRI at the discharge is reported below the reactors and is $2.7 \%$ and $3.7 \%$ respectively for ES and SSC, in good agreement with the plant data. 


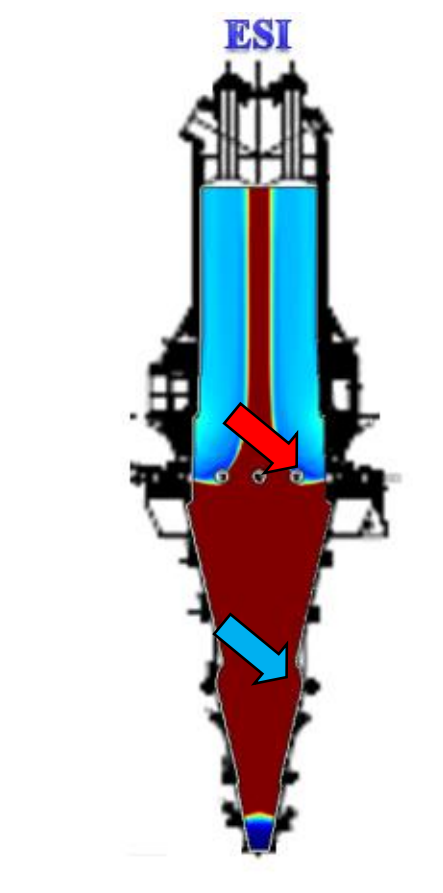

\section{A}

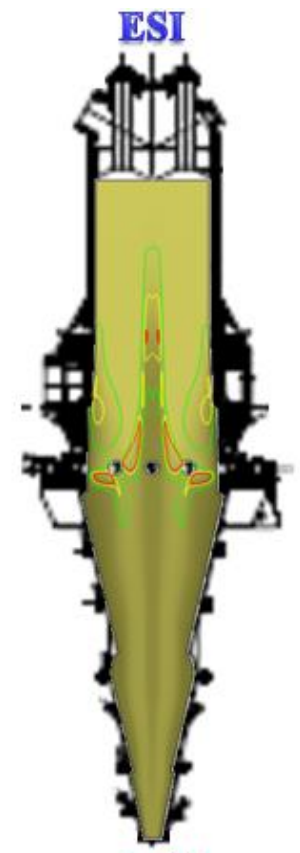

$2.7 \%$

\section{B}
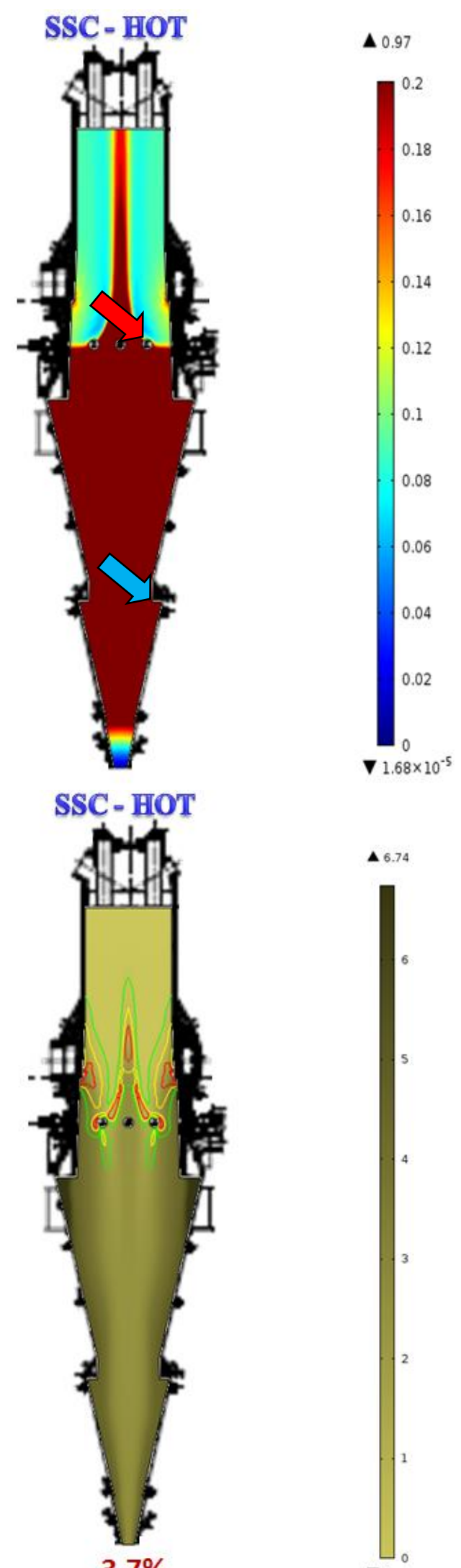

$3.7 \%$

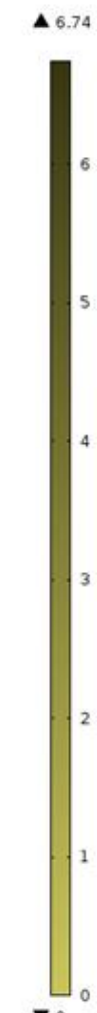

Figure 5. ENERGIRON Direct Reduction Reactor, with external reformer (ESI) and in ZR (SSC, simulated in Hot-discharge). A) Methane distribution along reactors (red arrows represent bustle gas injections; blue arrows stand for natural gas feeding point). B) Carbon maps and cracking reaction contours (green: low, yellow: middle, red: high). 
In ZR plants, the carbon deposition rate can be controlled by adding steam to the reducing gas fed to the Reactor which promotes the re-gasification of deposited carbon giving back $\mathrm{CO}$ and $\mathrm{H} 2$. It is easy to note that combining the first and third equation, the steam reforming reaction is obtained, meaning that a proper steam control in the reducing gas allows controlling the carburization and promoting the reforming. Data shown on Figure 2, collected during commissioning of Suez Steel Company ZR plant, confirms that ranging water percentage in the process gas from $4.5 \%$ up to $6.5 \%$, carbon content varies from $4.2 \%$ up to $2.6 \%$.

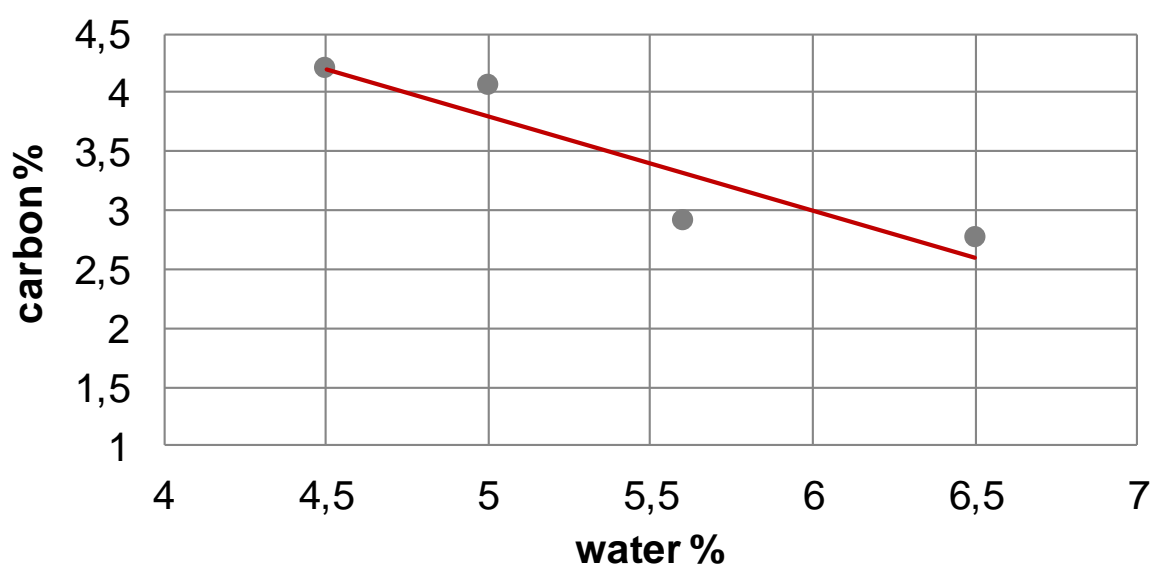

Figure 6. SSC ENERGIRON DRP plant data $-C$ content in the DRI vs water content in the $P G$ to reduction

As a consequence properly setting the natural gas fed to the reduction circuit, mainly controlling natural to reactor cone, together with the water content in the process gas, the percentage of carbon in the final DRI is controlled.

High quality DRI charged at the EAF at high temperature

Together with the chemical composition, that is mainly the carbon content and the degree of metallization, the other crucial characteristic of the DRI which sensitively affects the meltshop's performance is the temperature at which the DRI reaches the EAF. As previously anticipated, with the HYTEMP system, the thermal energy acquired by the iron ore pellet when converted in DRI is directly transferred from the reduction furnace to the EAF where is completely recovered promoting a reduction of the electrical energy consumption and tap-to-tap time, as well as lower refractory and electrodes consumption.

For example in Emirates Steel Industry Phase 1\&2 plants, with their typical EAF feed mix $(90 \%$ hot $+10 \%$ cold DRI), it is possible to save about $26 \mathrm{kWh} / \mathrm{tls}$ for every 100 ${ }^{\circ} \mathrm{C}$ increase in the DRI temperature, as shown in Figure where data collected with $\mathrm{DRI}$ at different degree of metallization are shown.

Together with the savings in electricity, also the EAF productivity increases with the DRI temperature. The tap-to-tap time decreases about $5 \%$ and the productivity increases accordingly per every $100^{\circ} \mathrm{C}$ increase in the DRI temperature (Figure). 


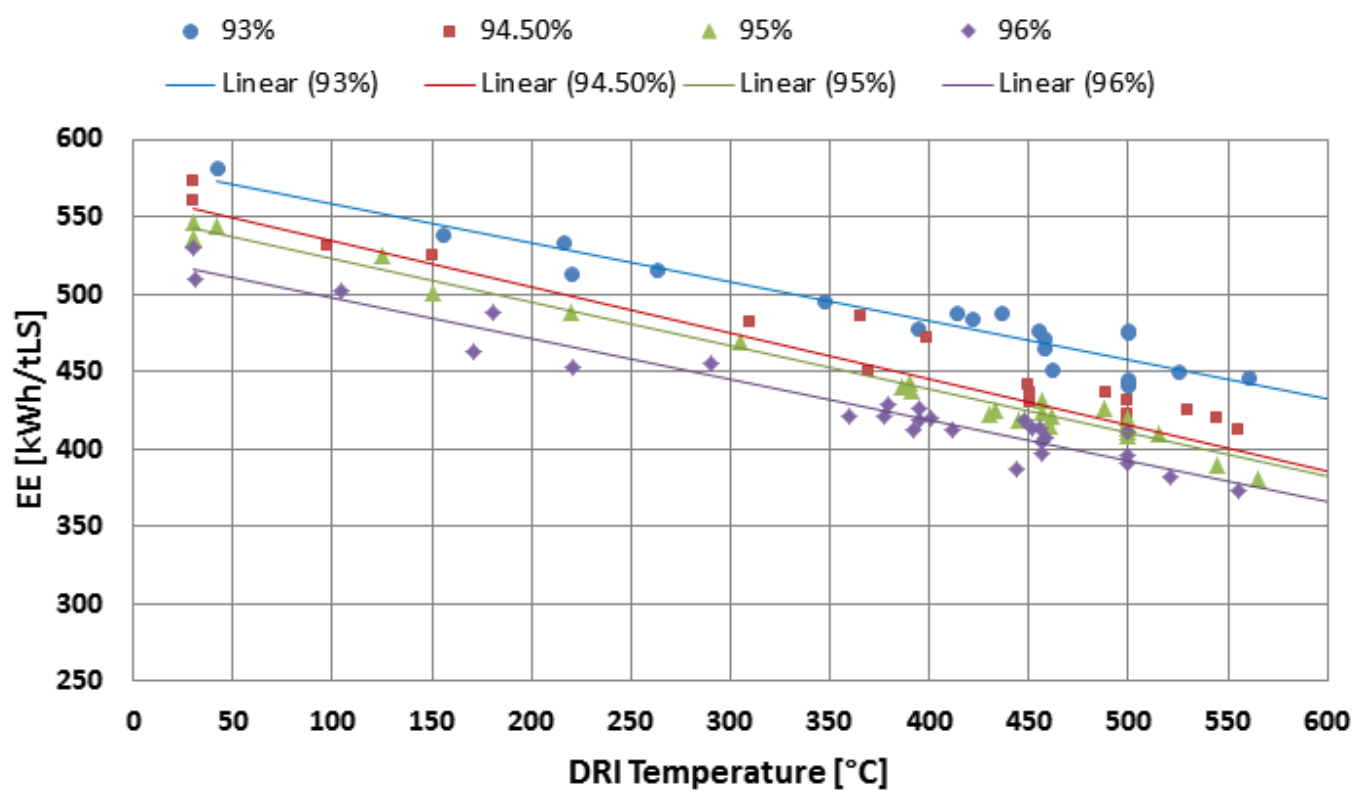

Figure 7. Effect of the DRI temperature (C\% in the range 2.0-2.5) on the EAF Electrical Energy Consumption

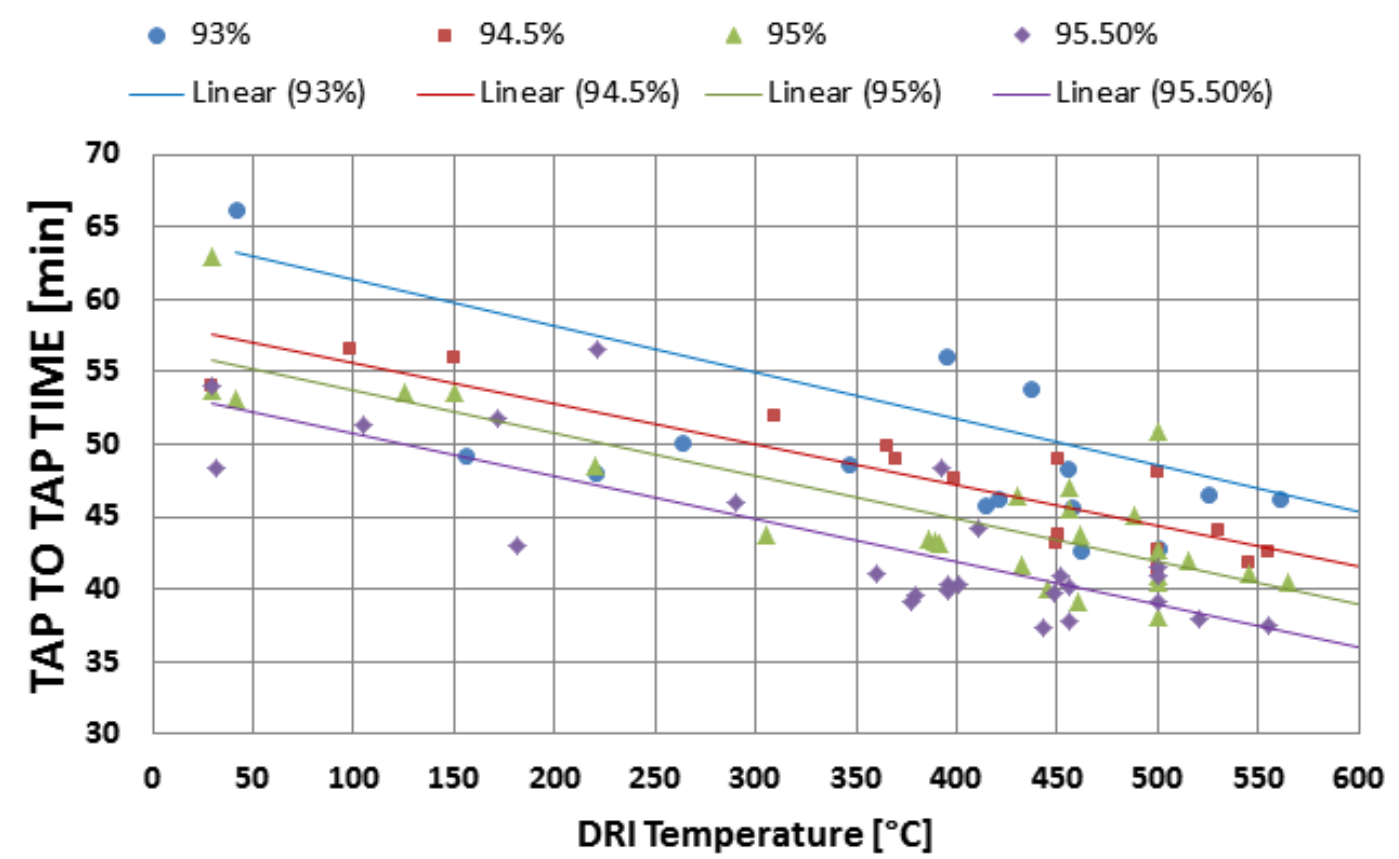

Figure 8. Effect of the DRI (C\% in the range 2.2-2.5) temperature and degree of metallization on the EAF Tap-to-Tap Time

A further improvement related to the temperature of DRI at the EAF has been obtained with the installation of the innovative lining of the reactor cone. This lining has been defined after a deep research dedicated to find the perfect material for not affecting the solid flow of DRI in the cone and, at the same time, not requiring modifications in volume and geometry of an already existing and proved cone. For this reason a refractory material with friction coefficient similar to the steel at low temperature has been designed and properly installed. With this modification the heat losses, due to heat transfer through the water jackets, have been significantly reduced resulting in higher DRI temperature directly at the Reactor discharge, less 
energy consumption and less make up water thanks to a reduced amount of sensible heat removed.

With this configuration, installed in the Suez Steel Reactor cone, DRI has been fed at the EAF at temperatures above $600^{\circ} \mathrm{C}$ as proved by plant data collected during the SSC DRP commissioning, completed at the end of 2014, shown in Figure.

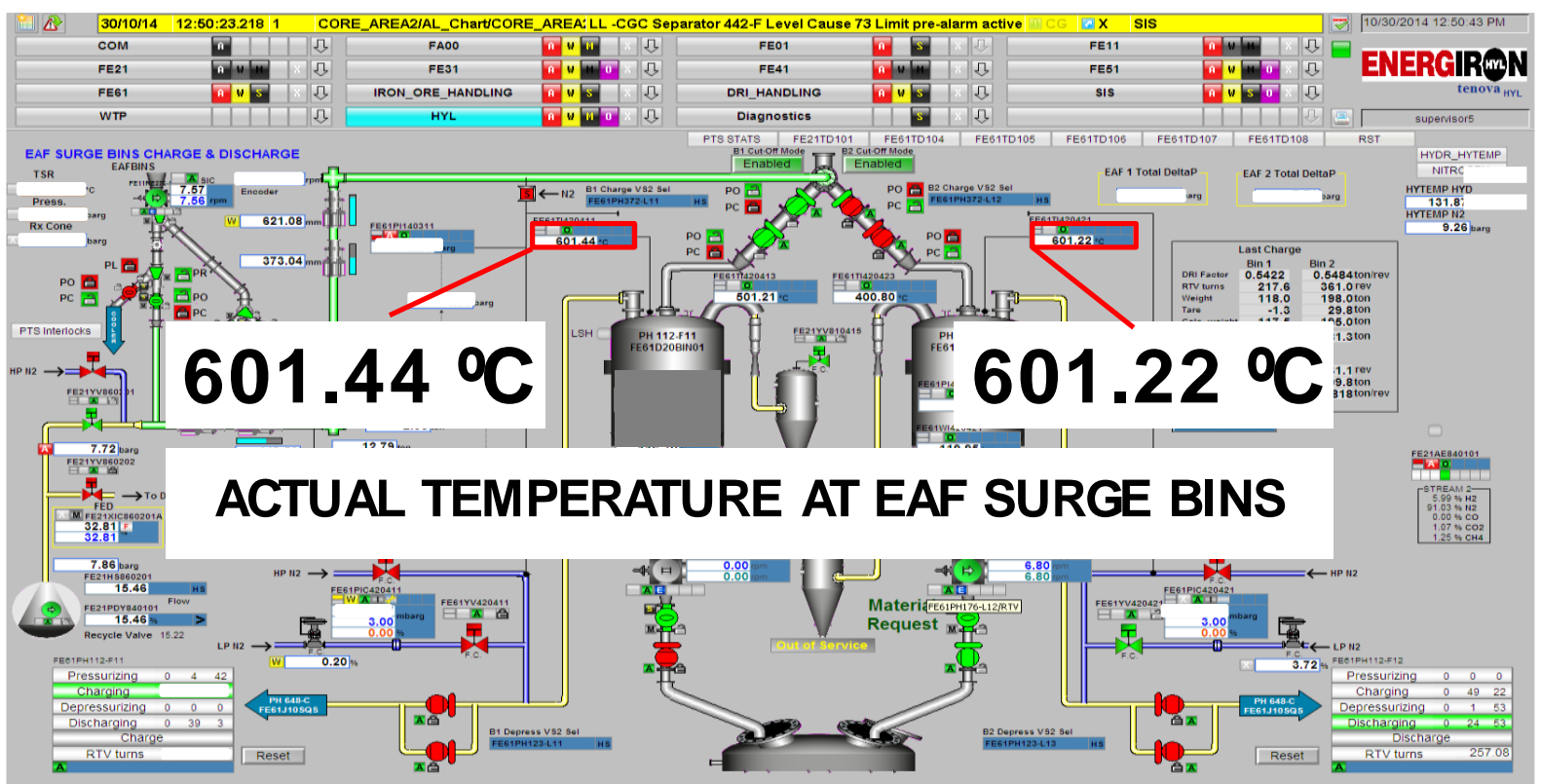

Figure 9. DRI temperature at EAF measured using thermocouples installed in the EAF Surge Bins.

This remarkable achievement has been recorded also during the Performance Test of Suez Steel DRP, carried out in a period of five days specifically from 30th October 2014 up to 4th November 2014. During these tests the plant capacity, as well as all the consumptions and quality parameters have been reached, confirming the excellent performance of this Energiron ZR plant (all data are shown in Table 1).

Table1. SSC Energiron DRP Performance Guarantee Test

\begin{tabular}{|l|c|c|c|c|}
\hline & Unit & \multicolumn{1}{c|}{$\begin{array}{c}\text { Achieved } \\
\text { results }\end{array}$} & \multicolumn{2}{|c|}{ Target } \\
\hline PRODUCTION & tons & 29262 & 29250 & $\mathrm{~min}$ \\
\hline AVERAGE METALLIZATION & $\%$ & 94.28 & 94 & $\mathrm{~min}$ \\
\hline AVERAGE CARBON CONTENT & $\%$ & 3.52 & 3.5 & $\mathrm{~min}$ \\
\hline AVERAGE NATURAL GAS CONSUMPTION & Net Gcal/t of DRI & 2.40 & 2.42 & max \\
\hline AVERAGE ELECTRICITY CONSUMPTION & Net Gcal/t of DRI & 93.17 & 95 & max \\
\hline AVERAGE HOT DRI TEMPERATURE & ${ }^{\circ} \mathrm{C}$ & 600 & 600 & min \\
\hline $\begin{array}{l}\text { AVERAGE IRON OXIDE PELLETS (on dry } \\
\text { basis and screened) }\end{array}$ & t of iron ore/t of DRI & 1.38 & 1.40 & max \\
\hline
\end{tabular}

Ultimately the benefits of continuous charging of $100 \%$ Hot DRI in the EAF has been confirmed also with the SSC EAF performance test carried out on November 2014 
(19-20 November 2014) when the achieved parameters, shown in Table 3, have been within the target ones once again.

Table 2. SSC, EAF performance tests parameters

\begin{tabular}{|l|c|c|c|}
\hline & units & HEATS & CONTRACT \\
& & $\mathbf{1 0 0} \%$ hot DRI & \\
\hline Tap to Tap & $\mathrm{min}$ & 52.3 & 54 \\
\hline El. Energy consumption & $\mathrm{kWh} / \mathrm{ls}$ & 379 & 395 \\
\hline Electrode consumption & $\mathrm{Kg} / \mathrm{tls}$ & $\mathrm{N} / \mathrm{A}$ & 1.34 \\
\hline Oxygen consumption & $\mathrm{Nm} 3 / \mathrm{ts}$ & 30.2 & 39.5 \\
\hline EAF process yield & $\%$ & 86.7 & 87.3 \\
\hline
\end{tabular}

\section{CONCLUSION}

The extreme flexibility of Energiron plants allows guaranteeing a very good product quality: easily controlling the carbon content and the temperature of the DRI fed at the EAF, all meltshop requirements can be fulfilled.

In this contents, in addition to the excellent results collected by plants in operations since 2011 (Emirates Steel plant), the completion of the Suez Steel Company integrated plant commissioning; another milestone in the steel industry world has been placed. Thanks to the innovative Energiron ZR technology and its perfect integration with a suitably designed EAF the DRI is available, at the EAF, both at high temperature (above $600^{\circ} \mathrm{C}$ at the EAF) and also at high carbon content (higher than $3.5 \%)$.

\section{REFERENCES}

1 N. Towhidi, J. Szekely. The influence of Carbon Deposition on the Reduction Kinetics of Commercial Grade Hematite Pellets with $\mathrm{CO}, \mathrm{H} 2$ and N2, Metallurgical Transactions B, Vol. 14B, 1983, 359.

2 H. J. Grabke, Carburization, carbide formation, metal dusting, coking, Materiali in technologije, 36, 2002, 6.

3 A. Schneider and J. Grabke, Effect of H2S on metal dusting of iron, Materials and Corrosion, 54, 2003, 793.

4 D. Dalle Nogare, A. Zugliano, A. Primavera, T. Melchiori, P. Canu, Multiphysics simulation of a DRP shaft furnace, Proceedings of the 5th SteelSIM, Ostrava 2013.

5 Energiron DR Plants Data kindly conceded by SSC (Suez Steel Company) and ES (Emirates Steel). 